Original article (short paper)

\title{
Neuromuscular control strategies of the trunk antagonist muscles during the Biering-Sorensen test in individuals with recurrent low back pain and healthy subjects
}

\author{
Ângela Kazue Morita \\ Nise Ribeiro Marques \\ Marcelo Tavella Navega \\ Universidade Estadual Paulista “Júlio de Mesquita Filho”, Marília, SP, Brasil
}

\begin{abstract}
Objectives: To compare the activation pattern of the trunk antagonistic muscles and also the myoelectric manifestations of muscle fatigue between subjects with and without recurrent non-specific low back pain, during the fatigue provocation of the erector spinae. Methods: The study involved 19 subjects with recurrent low back pain with a non-specific cause (seven men, 12 women, $38.53 \pm 8.12$ years, $68.35 \pm 18.12 \mathrm{~kg}, 1.66 \pm 0.09 \mathrm{~m}$ ), and 19 healthy subjects (seven men, 12 women, $40.42 \pm 8.63$ years, $69.57 \pm 12.76 \mathrm{~kg}, 1.64 \pm 0.07 \mathrm{~m}$ ). The electromyographic signal of the internal oblique, lumbar multifidus, rectus abdominis and lumbar iliocostalis muscles, bilateral, were collected during Biering-Sorensen test execution. Results: The group with low back pain showed a lower co-contraction rate of the internal oblique/lumbar multifidus $(p=0.006)$ and lower activation amplitude of the internal oblique $(p=0.019)$, both on the right side when compared to the group without low back pain. No differences were observed between the groups for muscle fatigue indicators $(\mathrm{p}>0.05)$. Conclusion: When the erector spine muscle fatigue occurs - even when the groups were similar as to the ability of extensors muscles to resist fatigue - differences were found between subjects with and without low back pain regarding the recruitment pattern of the task antagonist muscle, because subjects with low back pain showed lesser activation and co-contraction in relation to the healthy group.
\end{abstract}

Key-works: muscle fatigue, abdominal muscles, electromyography, muscle contraction

\section{Introduction}

Intervertebral stabilization is led by three interdependent subsystems: muscular or active, articular or passive and neural ${ }^{1,2}$. The dysfunction of the subsystems, due to pain or injury, impairs the ability of the neuromuscular control process afferent information and executes appropriate motor responses. Instability can occur, and it is characterized by decreased articular stiffness ${ }^{3}$, which leads to occurrence of excessive intervertebral movements and even greater damage to neural structures and passive components ${ }^{2,4}$. The consequences of instability make necessary a compensatory increase in trunk muscle co-contraction ${ }^{4}$.

The co-contraction is defined as the deliberate and simultaneous activation of two antagonistic muscle groups in order to stabilize the joint ${ }^{5}$. In a healthy control system, the strategy of increasing the agonist-antagonist trunk muscles contraction is not necessary in all tasks 6 . Previous studies of the neuromuscular activation pattern found greater co-contraction of the flexor and extensor lumbar muscles ${ }^{7-9}$, and greater trunk stiffness coefficient ${ }^{10}$ in people with low back pain when performing different tasks, as compared to healthy subjects. One study observed increases in spinal stiffness and trunk muscle activation when low back pain is elicited in healthy subjects and provided empirical evidence about the mediation of muscle activity in the spinal stiffness modification ${ }^{11}$. This adaptive strategy aims to restrict the range of trunk movement to avoid worsening or onset of pain and other injuries ${ }^{1,9,12}$.
In addition to muscle activation changes, some studies suggest that the cause of low back pain may be related to lower muscular endurance of the erector spinae, as subjects with low back pain have early manifestations of muscle fatigue compared to healthy people, when submitted to endurance tests ${ }^{13,14}$. The Biering-Sorensen test is widely used and is considered one of the most appropriate for clinical populations, in addition to being of low cost and easy execution ${ }^{13-15}$.

Muscle fatigue can change the neuromuscular control mechanism and affect the stiffness produced by the active contraction of the muscles that support the column ${ }^{1}$; or a constant hyperactivation of the trunk muscles, due to increased co-contraction, could also be the cause of least resistance in the erector spinae ${ }^{8}$. These changes may contribute to the recurrence of low back pain, as even in asymptomatic patients the co-contraction levels are not normalized ${ }^{7,8}$.

In order to corroborate new evidence about the possible relationship between muscular endurance of the erector spinae and trunk co-contraction modulation, the present study aimed to compare, between individuals with and without low back pain, the activation pattern of trunk antagonists muscles, caused by erector spinae fatigue, as well as to compare the myoelectric manifestations of muscle fatigue. It was expected that the muscle fatigue development would increase the co-contraction and activation levels of trunk antagonistic muscles in the low back pain group, and that this same group would be less resistant regarding the erector spinae fatigue, when compared to healthy subjects. 


\section{Methods}

\subsection{Subjects}

This study was approved by the local Ethics Committee in Research (process number: 0948/2014) and all participants signed the informed consent.

Thirty-eight sedentary individuals participated in this research, divided into control group (CG), formed by participants without low back pain, and experimental group (EG), composed of participants with recurrent non-specific low back pain. The sample size was determined based on pilot study data (effect size $=0.90$, power $=0.85$ and $\alpha$-error $=0.05$ ). Both groups had the same number of men (7) and women (12). The samples were homogeneous in terms of anthropometric characteristics (Table 1). All participants were recruited from within a university and a community medical school. To participate in the study, the volunteers could not present with a specific cause of their back pain, obesity (BMI $\geq$ $30 \mathrm{~kg} . \mathrm{m}^{-2}$ ), vertebral deformities that have led to function loss, history of back loss, neuromuscular or joint disease, current pregnancy or childbirth in the 6 months prior to study participation, or the presence of other chronic painful conditions. The EG was composed of participants who reported at least two episodes of low back pain in the last three months and were apt to perform the tests. Individuals who had not experienced lower back pain in the previous 12 months were included in the $\mathrm{CG}$.

Table 1. Antrophometric data (mean and standard deviation).

\begin{tabular}{lllc}
\hline \multicolumn{1}{c}{ Variables } & \multicolumn{1}{c}{ CG $(\mathbf{n}=\mathbf{1 9})$} & \multicolumn{1}{c}{ EG $(\mathbf{n}=\mathbf{1 9})$} & \multicolumn{1}{c}{$\mathbf{p}$} \\
\hline Age (years) & $40.42(8.63)$ & $38.53(8.12)$ & 0.491 \\
Body mass (kg) & $69.57(12.76)$ & $68.35(12.18)$ & 0.765 \\
Height (m) & $1.64(0.07)$ & $1.66(0.09)$ & 0.370 \\
BMI (kg.m $\left.{ }^{-2}\right)$ & $25.74(3.87)$ & $24.52(3.18)$ & 0.296 \\
\hline
\end{tabular}

$\mathrm{CG}$, control group; EG, experimental group; BMI, body mass index.

\subsection{Procedures}

Initially, the data regarding the characteristics of the painful lumbar symptom of the EG were collected. The pain intensity was measured by visual analog scale (VAS), where $0 \mathrm{~cm}$ represents no pain and $10 \mathrm{~cm}$, the worst possible pain ${ }^{7}$. After, in the same group, the Rolland Morris ${ }^{7}$ and the Fear Avoidance Beliefs Questionnaire (FABQ) ${ }^{16}$ were applied. This last questionnaire approaches the behavioral-cognitive aspects, such as fear and avoidance behaviors of low back pain subjects in relation to physical activity and work. In both questionnaires, higher scores indicate worse outcomes of the analyzed areas ${ }^{7,16}$.

The results indicated that subjects of EG had experienced painful dysfunction for the previous $9.11 \pm 7.83$ years (range 1-25 years), and among these, $15.78 \%$ required medical care and $26.31 \%$ had medication because of low back pain. The mean VAS was $2.23 \pm 2.86 \mathrm{~cm}$, while the Rolland Morris Questionnaire score was $3.21 \pm 2.55$ points, which is $13.37 \%$ of the maximum score. The FABQ work subscale was $10.78 \pm 10.17$ points and physical activity subscale was $26.10 \pm 8.38$ points.
Next, the electromyographic signals from both groups were obtained during maximal voluntary isometric contraction (MVIC) and the Biering-Sorensen test. All participants were familiarized with the execution of these procedures.

\subsubsection{Electromyography}

The participant skin surface was prepared to reduce skin impedance, by shaving and cleaning with alcohol application using a gauze ${ }^{17}$.

Double rectangular surface and adhesive electrodes $(\mathrm{Ag} /$ $\mathrm{AgCl}$ ) (3M Brazil, Sumaré, BRA), with an area of $1 \mathrm{~cm}^{2}$ and an inter-electrode distance of $2 \mathrm{~cm}$, were placed bilaterally along the fibers of the muscles: internal oblique (IO) ${ }^{18}$, upper fibers of the rectus abdominis (RA) ${ }^{1}$, multifidus (MU) ${ }^{17}$ and iliocostalis lumbar (IL) ${ }^{17}$.

Biological signals were obtained through the electromyography EMG 830C model (EMG System do Brasil, São José dos Campos, BRA), with eight channels and EMG Lab software (EMG System do Brazil, São José dos Campos, BRA), programmed with frequency sampling of $2000 \mathrm{~Hz}$ and total gain of 2000 times (20 times in the electrode and 100 times in the equipment). The common mode rejection ratio of the equipment was $>100 \mathrm{~dB}$, the system impedance was equal to $109 \mathrm{Ohms}$ and noise ratio of the signal was lower than $3 \mu \mathrm{V}$.

\subsubsection{Maximal voluntary isometric contraction}

The MVICs of trunk flexors, extensors and rotators were each repeated three times, for 4 seconds, with 2 minutes rest between the trials ${ }^{19}$. The flexors and rotators trunk muscles were tested in the sitting position on a chair adapted for the test (Figure 1). The extensor group was tested in the prone position, with the pelvis and lower limbs fixed by straps on an exercise bench. In all tests, the generation of muscular strength was resisted by a strap fixed on the trunk and the participants were verbally encouraged.

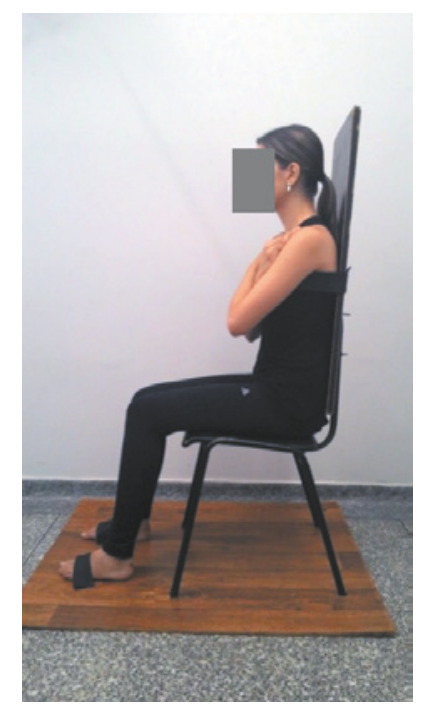

Figure 1. Chair adapted to the maximal isometric voluntary contraction test. 


\subsubsection{Biering-Sorensen test}

The subjects were laid in the prone position, with the cranial border of the iliac crest positioned at the upper edge of a timber support. The pelvis and lower limbs were fixed by straps ${ }^{13}$. Pillows were used under the iliac spines and legs to improve comfort. When starting the test, participants were instructed to cross the upper limbs in front of the chest and perform the extension of the trunk until the maximum comfortable amplitude (Figure 2) ${ }^{14}$. The participants were verbally encouraged to keep the trunk unsupported as long as possible, while the electromyographic signal and the time test were recorded.

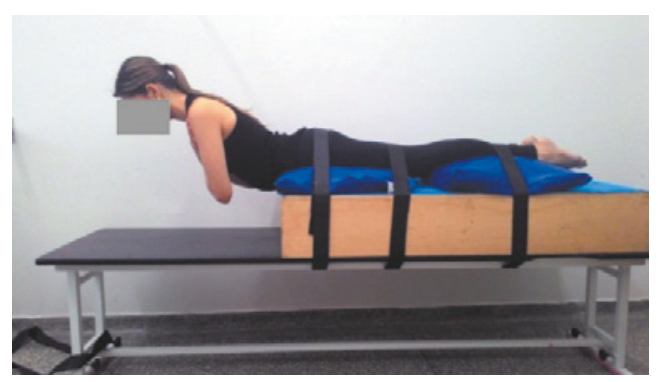

Figure 2. Execution of the Biering-Sorensen test.

\subsection{Data analysis}

The electromyographic signal was processed using Matlab (Mathworks ${ }^{\circledR}$, Natick, USA), in which was applied the Butterworth band-pass filter of the $20-500 \mathrm{~Hz}$ and $60 \mathrm{~Hz}$ notch filter. The analysis of the electromyographic signal was fulfilled in the time domain through the root mean square (RMS) value, and in the frequency domain, based on median frequency (MF) using the Fast Fourier Transformation. Sliding windows of $1 \mathrm{~s}$, with $0.5 \mathrm{~s}$ overlap, were used to calculate the RMS and MF. The RMS values were normalized by the MVIC values.

Muscle fatigue was analyzed by the MF slope, which is the linear regression coefficient of the MF values obtained during the Biering-Sorensen test.

The percentage of co-contraction was calculated using the following equation:

$$
\text { Co-contraction } \%=\frac{2 \times \text { common area A \& B } \times 100}{\mathrm{~A} \text { area }+\mathrm{B} \text { area }}
$$

Where Co-contraction \% is the percentage of co-contraction between two antagonistic muscles; area $\mathrm{A}$ is the smoothed curve of muscle $A$; area $B$ is the smoothed curve of muscle $\mathrm{B}$; common area between $\mathrm{A} \& \mathrm{~B}$ is the common curve of the muscle $\mathrm{A}$ and muscle $\mathrm{B}^{20}$. To obtain the smoothed curve, the electromyographic signal was rectified by full-wave method and smoothed using a Butterworth low pass filter of the 4th order, at $6 \mathrm{~Hz}$ of the cutoff frequency.

The pairs of antagonistic muscles were composed according to its function. The following pairs were analyzed together: MU and IO, because were considered trunk deep stabilizers; IL and RA because they are superficial muscles and perform dynamic function ${ }^{12}$.

\subsection{Statistical analysis}

Statistical analysis was performed using PASW Statistics 18 (SPSS inc.) package. Data normality was tested by the ShapiroWilk test. The normalized RMS, MF slope and performance time in the Biering-Sorensen test showed normal distribution and were analyzed using the Student t-test for independent samples, while the co-contraction rate showed non-normal distribution and was analyzed by the Mann-Whitney test. The level of significance was set at $\mathrm{p}<0.05$.

\section{Results}

\subsection{Fatigue test: performance and electromyographic variables.}

There was no significant difference between the groups for the maintenance time of Biering-Sorensen test $(\mathrm{p}=0.38)$. EG and CG reached, on average, $97.31 \mathrm{~s}$ (37.11s) and 111.68s (59.88s), respectively.

Significant differences were found between groups for the variables: IO normalized RMS (p = 0.019) (Figure 3 ) and IO/ MU co-contraction rate $(p=0.006)$ (Figure 4$)$, both on the right side, where CG had higher mean values compared to EG.

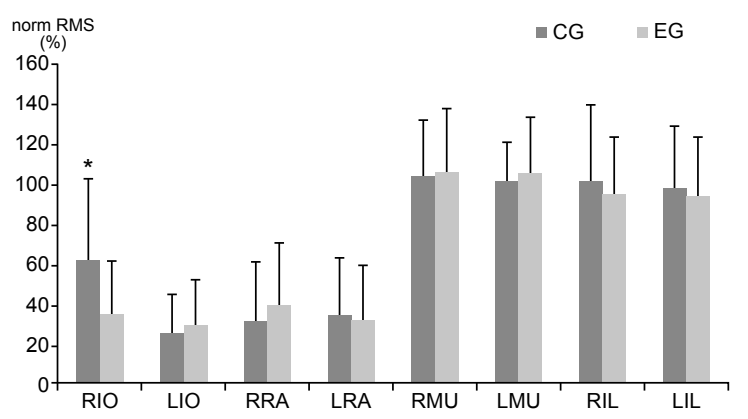

Figure 3. Comparison of the normalized RMS (\%) obtained during the Biering-Sorensen test (mean \pm standard deviation). $\mathrm{CG}$, control group; EG, experimental group; IO, internal oblique; RA, rectus abdominis; MU, lumbar multifidus; IL, iliocostalis lumbar; R, right; L, left; ${ }^{*} \mathrm{p}<0.05$ when comparing the groups.

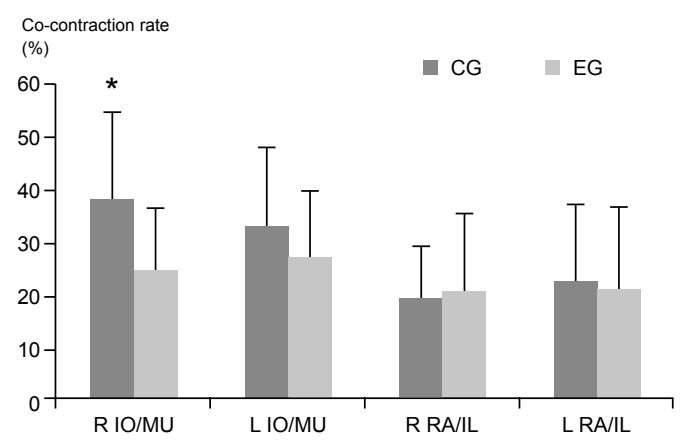

Figure 4. Comparison of the co-contraction rate (\%) obtained during the Biering-Sorensen test (mean \pm standard deviation). $\mathrm{CG}$, control group; EG, experimental group; IO, internal oblique; RA, rectus abdominis; MU, lumbar multifidus; IL, iliocostalis lumbar; R, right; L, left; ${ }^{*} \mathrm{p}<0.05$, when comparing the groups. 
There were no significant differences between groups for the MF behavior of the erector spinae (Table 2).

Table 2. Comparison of the median frequency slope coefficients obtained during the Biering-Sorensen test (mean and standard deviation).

\begin{tabular}{lccc}
\hline Muscle & CG & EG & p \\
\hline R MU & $-0.58(0.77)$ & $-0.93(0.83)$ & 0.184 \\
L MU & $-0.79(0.56)$ & $-0.58(0.51)$ & 0.211 \\
R IL & $-0.45(0.38)$ & $-0.41(0.51)$ & 0.825 \\
L IL & $-0.27(0.42)$ & $-0.51(0.45)$ & 0.099 \\
\hline
\end{tabular}

$\mathrm{CG}$, control group; EG, experimental group; MU, multifidus; IL, iliocostalis lumbar; R, right; L, left.

\section{Discussion}

The aims of this study were to analyze the activation pattern of antagonistic trunk muscles among healthy persons and subjects with recurrent non-specific low back pain, when localized muscle fatigue of the spine erector was induced, and to compare the myoelectric manifestations of muscle fatigue among these groups. The results showed that the low back pain group had less activation of right $\mathrm{IO}$, as well as lower $\mathrm{IO} / \mathrm{MU}$ co-contraction rate on the right side, when compared to healthy subjects. No significant differences were observed between the groups regarding the MF behavior (slope) of the trunk extensor muscles, while causing fatigue of this muscle group. The presented findings contradict the previously established hypotheses, as expected higher co-contraction of the analyzed muscles and lower muscular endurance of the erector spine in subjects with low back pain.

\subsection{Muscle fatigue of the spine erector}

Despite the premise that the erector spinae of subjects with low back pain are more susceptible to fatigue compared to healthy subjects $^{13,14}$, some authors ${ }^{15,21,22}$ that used similar methodology applied in our study also found no differences between groups with and without low back pain for the MF analysis.

The used evaluation protocols could have influenced the results. Studies that used protocols with additional load to the lumbar erector, relative to a percentage of the trunk extension MIVC, and pre-defined time test with up to 120 seconds $^{13}$, found a lower resistance in some lumbar erector muscle in the EG when compared with the control ${ }^{13,14}$. Protocols that considered exhaustion as interruption criteria for the Biering-Sorensen test, as used in this study, did not obtain differences between the groups for the electromyographic variable ${ }^{15,21}$.

Although the main reason for lower muscular endurance is based on the predominance of type II fibers in the erector spinae of the low back pain patients ${ }^{13}$, histomorphometry analysis revealed no differences between healthy and low back pain subjects regarding the proportion of the fibers number or the area occupied by the type I and II fibers ${ }^{15}$. In the present study, we selected the exhaustion criteria because the prolonged maintenance of the test position require increased activity of the type I fibers, characterized by being more resistant to localized muscle fatigue ${ }^{13}$.

It is suggested that subjects with recurrent low back pain and a non-specific cause, sedentary and with low scores for pain and functional disability are not differentiated from healthy subjects regarding the erector spinae ability to resist fatigue, when performing the Biering-Sorensen test until the exhaustion. It proposes that for this clinical population, the investigation of muscle fatigue using the Biering-Sorensen test would require an additional load against the erector spinae.

\subsection{Co-contraction of antagonistic trunk muscles}

The recurrent pain and tissue damage of the lumbar spine limit the participation of posterior structures of the trunk to provide stiffness, with the spine becoming unstable ${ }^{23}$. For tasks that do not involve muscle fatigue provocation, low back pain subjects are characterized by adopting the compensatory mechanism through increasing the activation and muscular co-contraction of the trunk at higher levels than healthy subjects ${ }^{7-9}$. The task performed in this study, which involved the fatigue provocation of muscles located in pain area, did not cause the same compensation, whereas the increases in co-contraction and antagonist activation was lower in the EG compared to the healthy group.

However the antagonist muscle activation produces forces in the opposite direction of the agonist muscles, which seems to work against the agonist action and impair the endurance time of task support, a minimal amount of antagonist activation is required to optimize the torque output of the agonists. For this purpose, in healthy subjects, co-contraction is modulated constantly by the central nervous system to balance the opposites forces generated by the agonists and antagonists trunk muscles throughout the task, in order to facilitate their execution ${ }^{5}$.

These results regarding the increase in antagonist activation in CG corroborate with the Granata and Slota findings ${ }^{1}$. The authors found that when fatigue of erector spine is caused by successive weight lifting, there was a significant increase in electromyography activity of abdominal muscles. Fatigue could change the trunk stiffness and impair the extensor group ability to stabilizing the spine. If fatigue is not severe, the compensatory antagonist recruitment would be enough to restore the stability. Although this mechanism contributes to increase vertebral compression load and increases the risk of injury, in intact systems concomitant increase in stability is greater than the overload produced on the column ${ }^{24}$.

Regarding the findings of the IO, evidence shows that the transversus abdominis and the lower portion of the IO stabilize the lumbar spine and pelvis through the tensioning of the thoracolumbar fascia and increase in intra-abdominal pressure, so the spine becomes stiffened ${ }^{18,25,26}$. The thoracolumbar fascia joins the aponeurotic sheaths of deep abdominal muscles with the sheath that surrounds the MU, IL and longissimus ${ }^{25}$. The anatomical junction of IO and MU could justify the higher co-contraction of these muscles and, despite the difference is significant between groups only on the right side, the co-contraction of the left side was also higher in the CG. 
A further argument is based on the modulation of muscle activation in response to task demand. In activities that require submaximal efforts, as applied in the test, the recruitment of the deep abdominal muscles is enough to provide stability and only those activities that require maximum muscle strength require the recruitment of superficial muscles ${ }^{6}$.

In relation to low back pain population, many studies show changes in the activation pattern of the deep abdominal muscles, such as delay in onset ${ }^{18}$, less automatic activation in unstable positions ${ }^{12,26}$ or postural changes ${ }^{27}$ compared to healthy subjects, besides clinical improvement when training the selective contraction of these muscles ${ }^{28}$. One study found better results in excessive lumbar vertebrae translation and rotation, analyzed by x-rays images, in subjects with chronic low back pain that trained co-contraction of the deep stabilizers muscles of the trunk, when compared with the general exercises group ${ }^{29}$. The possible impairment of the isolated activation of the deep abdominal muscles could be caused by the general increase in co-contraction of the trunk muscles, which would affect the selective muscular recruitment ${ }^{4}$.

The decrease in antagonist activation was also found when provoking the immediate spinal instability, through the static stretching of the posterior trunk structures for 10 minutes. Before and immediately after the stretching, the participants performed submaximal isometric tasks of the trunk flexors and extensors, each for $10 \mathrm{~s}$, and this study found a decrease in antagonist activation, in both tasks, after stretching ${ }^{23}$. In situations of chronic instability, this mechanism would be adopted in tasks that require muscular endurance by longer period of time.

The results of this study contribute evidence indicating that the strategies selected by the neuromuscular control, in order to regulate the stiffness of the trunk, are differentiated in the presence of low back pain and that these strategies are not fixed, and instead, vary with task characteristics. According to some authors ${ }^{24}$, the increase of antagonist activation can be determined by the risk of injury, in that high overload activities lead to a decrease of coactivation in order to reduce the additional load produced by muscle contraction. In this context, the Biering-Sorensen test could impose subjects with low back pain sufficient overload, in which increasing activation of the antagonist muscle would be advantageous.

\section{Limitations}

This study did not consider the localization of low back pain. This would require larger samples of subjects with bilateral and unilateral low back pain for a more specific analysis. Furthermore, there was no control of trunk rotation during the test execution, which could have resulted in unilateral differences. It is suggested that future studies include the analysis of lower limb muscles that can contribute to performance in the Biering-Sorensen test.

\section{Conclusion}

The results of this study showed that during the evaluation test of erector spinae endurance, subjects with low back pain had less activation of $\mathrm{IO}$ and lower rate of $\mathrm{IO} / \mathrm{MU}$ co-contraction, observed on the right-hand side, compared to healthy subjects. The demand of the Biering-Sorensen test until exhaustion necessitated the recruitment of IO in intact neuromuscular control systems, while this strategy was not observed in EG. Furthermore, muscle fatigue parameters showed no differences between the groups with and without low back pain, which could indicate that both groups are similar regarding the ability of the erector spinae to resist the fatigue, when performing the Biering-Sorensen test until the exhaustion.

\section{References}

1. Granata KP, Slota GP. Influence of fatigue in neuromuscular control of spinal stability. Hum Factors. 2004;46(1):81-91.

2. Panjabi MM. The stabilizing System of the spine. Part I. Function, dysfuntion, adaptation, and enhancement. J Spinal Disord. 1992;5(4):383-9.

3. Izzo R, Guarnieri G, Guglielmi G, Muto M. Biomechanics of the spine. Part I: Spinal stability. Eur J Radiol. 2013;82(1):118-26.

4. Van Dieen JH, Selen LPJ, Cholewicki J. Trunk muscle activation on low-back pain patients, an analysis of the literature. $\mathrm{J}$ Electromyogr Kinesiol. 2003;13(4):333-51.

5. Duchateau J, Baudry S. The neural control of coactivation during fatiguing contractions revisited. J Electromyogr Kinesiol. 2014;24(6):780-8.

6. Mccook DT, Vicenzino B, Hodges PW. Activity of deep abdominal muscles increases during submaximal flexion and extension efforts but antagonist co-contraction remains unchanged. J Electromyogr Kinesiol. 2009;19(5):754-62.

7. Butler HL, Hubley-Kozey CL, Kozey JW. Changes in electromyographic activity of trunk muscles within the sub - acute phase for individuals deemed recovered from a low back injury. J Electromyogr Kinesiol. 2013;23(2):369-77.

8. D'Hooge R, Hodges P, Tsao H, Hall H, Macdonald D, Danneels L. Altered trunk muscle coordination during rapid trunk flexion in people in remission of recurrent low back pain. J Electromyogr Kinesiol. 2013;23(1):173-81.

9. Freddolini M, Strike S, Lee RYW. The role of trunk muscles in sitting balance control in people with low back pain. J Electromyogr Kinesiol. 2014;24(6):947-53.

10. Freddolini M, Strike S, Lee RYW. Stiffness properties of the trunk in people with low back pain. Hum Mov Sci. 2014;36:70-9.

11. Wong AY, Parent EC, Prasad N, Huang C, Chan KM, Kawchuk GN. Does experimental low back pain change posteroanterior lumbar spinal stiffness and trunk muscle activity? A randomized crossover study. Clin Biomech. 2016;34:45-52.

12. Ehsani F, Arab AM, Jaberzadeh S, Salavati M. Ultrasound measurement of deep and superficial abdominal muscles thickness during standing postural tasks in participants with and without chronic low back pain. Man Ther. 2016;23:98-105.

13. Tsuboi T, Satou T, Egawa K, Izumi Y, Miyazaki M. Spectral analysis of electromyogram in lumbar muscles: fatigue induced endurance contraction. Eur J Appl Physiol Occup Physiol. 1994;69(4):361-6. 
14. Candotti CT, Loss JF, Pressi AMS, Castro FAS, Torre ML, Melo $\mathrm{MO}$, et al. Electromyography for assessment of pain in low back muscles. Phys Ther. 2008;88(9):1061-7.

15. Crossman K, Mahon M, Watson PJ, Oldham JA, Cooper RG. Chronic low back pain-associated paraspinal muscle dysfunction is not the result of a constitutionally determined "adverse" fibertype composition. Spine. 2004;29(6);628-34.

16. Abreu AM, Faria CDCM, Cardos SMV, Teixeira-Salmela LF. Versão brasileira do Fear Avoidance Beliefs Questionnaire. Cad Saúde Pública. 2008;24(3):615-23.

17. Hermens HJ, Freriks B, Merletti R, Rau G, Disselhorst-Klug C, Stegeman DF, et al. Senian.org [Internet]. Netherlands: Project Management Office. Recommendations for sensor locations on individual muscles [about 2 screens]. Available from: http://www. seniam.org [Acessed 20th Sep 2013].

18. Massé-Alarie H, Flamand VH, Moffet H, Schneider C. Corticomotor control of deep abdominal muscles in chronic low back pain and anticipatory postural adjustments. Exp Brain Res. 2012;218(1):99-109.

19. Vera-Garcia FJ, Moreside JM, Mcgill SM. MVC techniques to normalize trunk muscle EMG in healthy women. J Electromyogr Kinesiol. 2010;20(1):10-6.

20. Candotti CT, Carvalho KV, La Torre M, Noll M, Varella M. Ativação e co-contração dos músculos gastrocnêmio e tibial anterior na marcha de mulheres utilizando diferentes alturas de saltos. Rev Bras Cienc Esporte. 2012;34(1);27-39.

21. Beneck GJ, Baker LL, Kulig K. Spectral analysis of EMG using intramuscular electrodes reveals non linear fatigability characteristics in persons with chronic low back pain. J Electromyogr Kinesiol. 2013;23(1):70-7.

22. Cai C, Kong PW. Low back and lower-limb muscle performance in male and female recreational runners with chronic low back pain. J Orthop Sports Phys Ther 2015; 45(6):436-43.

23. Lee N, Kang H, Shin G. Use of antagonist muscle EMG in the assessment of neuromuscular health of the low back. J Physiol Anthropol. 2015;34(1):6p.

24. Granata KP, Marras WS. Cost-benefit of muscle co-contraction in protecting against spinal instability. Spine. 2000;25(11):1398-404.
25. Willard FH, Vleeming A, Schuenke MD, Danneels L, Schleip R. The thoracolumbar fascia: anatomy, function and clinical considerations. J Anat. 2012;221(6):507-36.

26. Rasouli O, Arab AM, Amiri M, Jaberzadeh S. Ultrasound measurement of deep abdominal muscle activity in sitting positions with different stability levels in subjects with and without chronic low back pain. Man Ther. 2011;16:388-93.

27. Miura T, Yamanaka M, Ukishiro K, Tohyama H, Saito $\mathrm{H}$, Samukawa $\mathrm{M}$ et al. Individuals with chronic low back pain do not modulate the level of transversus abdominis muscle contraction across different postures. Man Ther. 2014;19(6):534-40.

28. Hwangbo G, Lee CW, Kim SG, Kim HS. The effects of trunk stability exercise and a combined exercise program on pain, flexibility, and static balance in chronic low back pain patients. $\mathrm{J}$ Phys Ther Sci. 2015;27(4):1153-5.

29. Javadian Y, Akbari M, Talebi G, Taghipour-Darzi M, Janmohammadi N. Caspian J Intern Med. Influence of core stability exercise on lumbar vertebral instability in patients presented with chroniclow back pain: A randomized clinical trial. 2015;6(2):98-102.

\section{Corresponding author}

Ângela Kazue Morita

Avenida Hygino Muzzi Filho, 737, Bairro: Mirante, Marília, SP, Brasil.

Email: angela.morita@yahoo.com.br

Manuscript received on April 24, 2016

Manuscript accepted on July 22, 2016

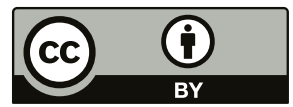

Motriz. The Journal of Physical Education. UNESP. Rio Claro, SP, Brazil - eISSN: 1980-6574 - under a license Creative Commons - Version 3.0 